\title{
IoT in Logistics- Smart Product Tracking Solution
}

\author{
Namboodiri Akash Prasad \\ Student, Department of Electronics and Communication \\ Amrita School of Engineering \\ Bengaluru, India \\ Pooja.S.Babu \\ Student, Department of Computer science and Engineering \\ The National Institute of Engineering \\ Mysore, India
}

\begin{abstract}
The paper proposes a less cumbersome and more efficient product tracking methodology. The existing method remotes the factory officials from access to real time information about the product. It requires that most of the details are updated manually. A more efficient design is a system which automates the whole process by eliminating the need for manual intervention. A convenient shift from a desktop to an app based ecosystem, promotes the hands-on-control aspect of the system. The paper tries to analyze and suggest a mechanism to smoothen the endto-end logistics of a B2B scenario. It aims at designing and implementing an end to end product tracking mechanism, using a Raspberry Pi as a central hub, RFIDs and IR sensors for product identification, An Android app supplements the smooth ordering process and live tracking the product using Google Map in the app. the system also includes ZigBee network that is designed to facilitate the automated parking solution for incoming and outgoing trucks and other vehicles
\end{abstract}

Keywords-Internet of Things, Industry 4.0, Raspberry Pi, RFID, ZigBee, IR Sensors, Cloud computing

\section{I.INTRODUCTION}

Logistics is the management of the flow of things between the origin and destination in order to meet requirements of customers. Industrial machinery can typically identify products through either Bar Code or RFID technologies. The unique product identification mechanism is responsible for advancement in the field of industrial automation. Industry 4.0 is a collective term for technologies and concepts of value chain organization. Industry 4.0 missionizes a "Smart Factory". The modular structured Smart Factories of Industry 4.0, encompass cyber-physical systems that monitor physical processes that co-exist in the factory, create a virtual image of the physical realms and make decentralized pronouncements. Over the Internet of Things, cyber-physical systems effectively connect with each other and the humans in real time. The Internet of Services provide internal and cross-organizational services and the values chain effectively uses the information gathered. The need of the hour is remodeling the industry to fit to the I4.0 standard, allowing it to establish all the design principles-interoperability, virtualization, decentralization, real-time capability, service orientation and modularity.

The main problem faced in the existing system is the manual entry and tracking of most the activity in the supply chain management and logistics. The technology has undergone fewer changes. Absence of real-time tracking of products and inefficient barcodes decrease the reliability of the system. Barcodes, due to the Line of Sight issues are quite cumbersome to work with. The solution presented in the paper hopes to address these issues by reducing the cost drastically, also making the whole process easier to work for the user.

The paper presents a proof of concept $(\mathrm{PoC})$ of an end-to-end supply chain management system. The Android app is used to order the products. RFID reader are connected to the Pi, read and tag the products. The products are loaded into a truck ( $\mathrm{RC})$, and are tracked using IR sensors embedded within the board of the PoC. Raspberry $\mathrm{Pi} 2 \mathrm{~B}+$ is used to push the data into the cloud. The live data can be accessed in real-time using the mobile app. A ZigBee network offers a parking solution for the vehicles entering the factory area.

\section{MOTIVATION}

\subsection{Scenario}


Assume a scenario in which a factory official (customer) needs to order certain quantities of certain products from the manufacturer of the product. The customer has to open his desktop, fill out the form, wait for the manufacturer to accept the order. Once, the product has been packaged and sent, he has to wait indefinitely, without knowing the actual location of his order.

This leads to wastage of time, energy and resources. He has no access to real time geo-location or the status of the product. He has to use a desktop and fill applications, while it can be done easily anytime and from anywhere using a mobile app.

\begin{tabular}{|c|c|}
\hline Component & Description \\
\hline Raspberry PI 2 B+ & $\begin{array}{c}2^{\text {nd }} \text { generation. 5V supply. } \\
\text { 1GB RAM. }\end{array}$ \\
\hline EM 18 RFID Reader & $\begin{array}{c}\text { Operational frequency: } 125 \\
\mathrm{KHz}\end{array}$ \\
\hline RFID Tags & $\begin{array}{c}\text { Operational frequency: } 125 \\
\mathrm{KHz}\end{array}$ \\
\hline XBee modules & Range: $100 \mathrm{~m}$ \\
\hline IR Sensors & Range: $10 \mathrm{~cm}$ \\
\hline Idle & Text editor for Python \\
\hline $\mathrm{X}-\mathrm{CTU}$ & Configure XBees \\
\hline Phant & Data logging live updates \\
\hline FireBase & $\begin{array}{l}\text { Updating live data and } \\
\text { interfacing with the app. }\end{array}$ \\
\hline
\end{tabular}

Table 1. Specification of the components

\subsection{Challenges faced currently}

1. Ever increasing human errors crafting huge loss.

2. Cumbersome tracking of the products, hiding the real-time information from the user.

3. Theft or damage of the products.

\section{PRINCIPLES AND BUILDING BLOCKS OF SMART PRODUCT TRACKING SOLUTION}

\subsection{Methodology}

The design consists an integrated hardware-software approach. The RFID is read using a reader. The reader transfers the ID number through the Serial port of the Raspberry Pi. Each RFID reader also transfers its respective location to the $\mathrm{Pi}$. The $\mathrm{Pi}$ concatenates the data with the timestamp. A Python script connects the 
Raspberry Pi with a server. The script uses protocol while connecting with the server. The server is updated every time the RFID detects an ID. The locations are mapped from the location in PoC to the actual location in Google Map.

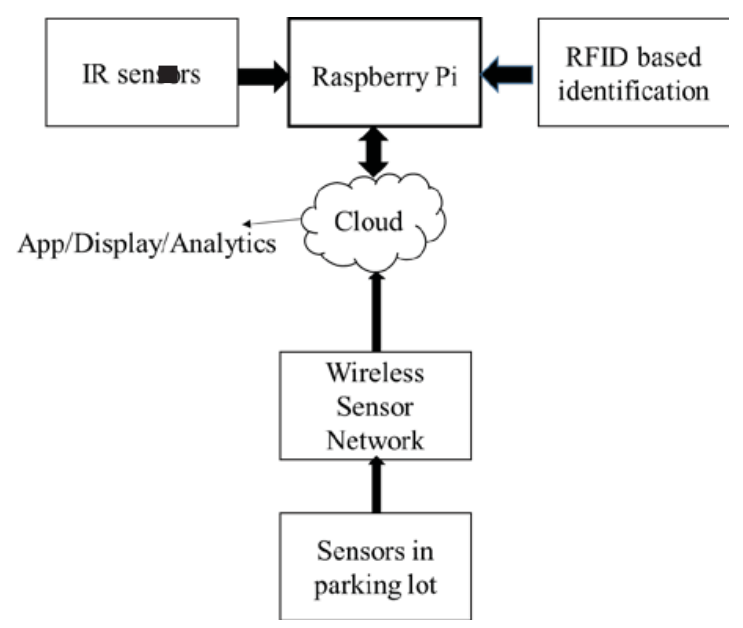

Figure 1. Architecture of Smart Product Tracking Solution

\subsection{Process Flow of the system}

i. The customer, first, logs in the app. The customer can place order of products and the quantity.

ii. Once the customer finalizes his cart, he can proceed to checkout.

iii. Once the customer places the order, the admin can approve/reject the order.

iv. Once the order is accepted, the algorithm finds out the best truck/vehicle for transporting the products according to the size, shape and quantity.

v. The products are given RFID tags. The tags are read at the gates of the factories and updated periodically. The products are now loaded into the truck ( $\mathrm{RC}$ truck).

vi. The truck is tracked by IR sensors. If the truck passes through a sensor, it will detect it, and the Pi will update the FireBase cloud. The app will fetch the data from the cloud and update the location accordingly in the map.

vii. The admin can always know the status of the product through Phant server.

viii. The customer is always updated via SMS regarding the status of their order.

ix. The RFID readers are located at each factories and it will read the tags which products are going in and out.

x. Every time the vehicle reaches a factory gate, it is assisted in parking by a ZigBee mesh, which will guide the driver to vacant parking spaces in order to save time and ensure quick delivery.

xi. Once the product is received, the admin and the customer are both alerted by SMS.

\section{PROTOTYPE}

\subsection{Prototype implementation}

Once the ordering has been done and the products have been dispatched, the following process takes places:

i. The truck ( $\mathrm{RC}$ truck) collects the products from Plant 1. The products' RFID are read using the RFID reader installed at the Plant's gate. The IDs are stored in the cloud

ii. After collecting the items, the truck proceeds further the road.

iii. At location 1, embedded IR sensors detect the vehicle and update is sent to the cloud server

iv. The truck reaches Plant 2. The products are unloaded and reloaded according to the demand. It follows the same procedure as in Plant 1.

v. Similarly, a location update is triggered at location 2. 
vi. Finally, the truck reaches the customer location. At the customer location, the vehicle is guided to appropriate parking slot, which functions using a ZigBee mesh network.

vii. Once the product reaches the customer, the customer is notified through SMS.

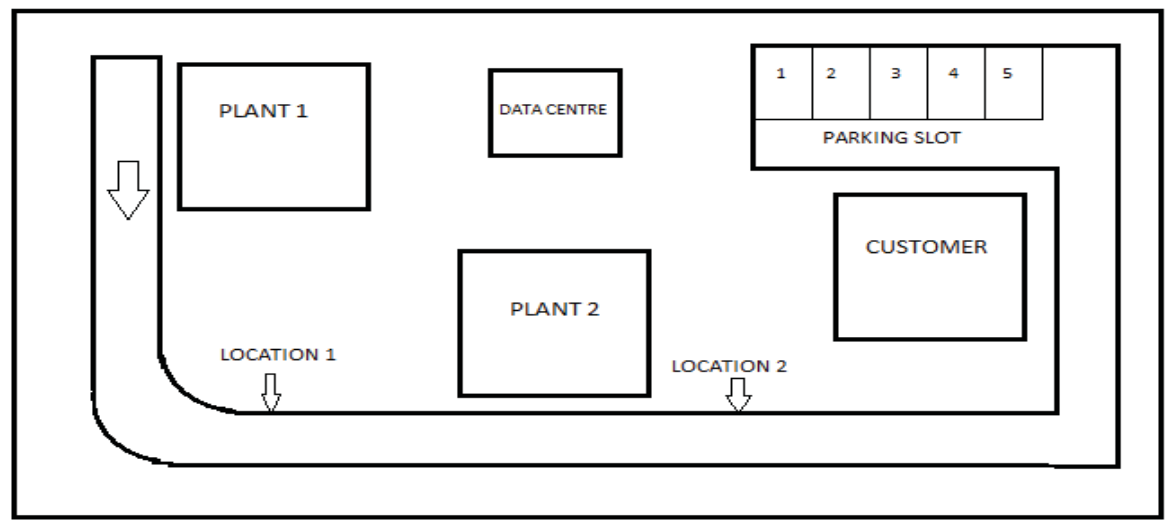

Figure 2. Bluprint of the model

\subsection{Results}

The results are evaluated on the ease of the operation and accessibility to the real time data. The following figures draw a clear explanation of the same.

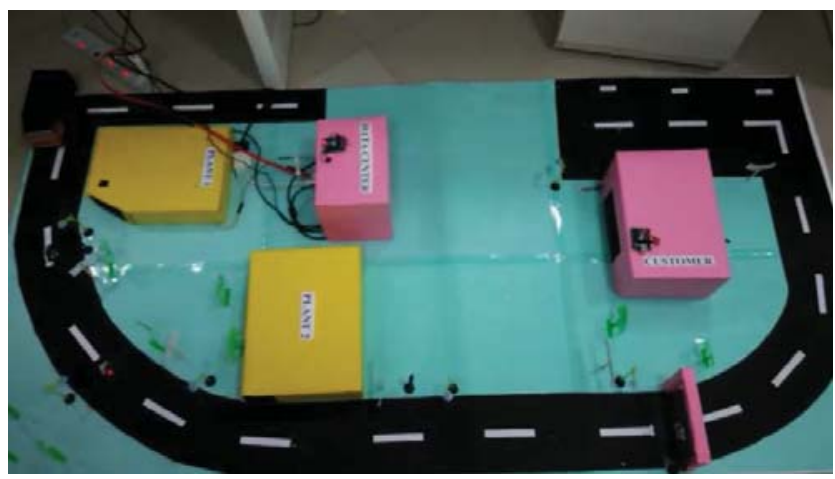

Figure 3. Bird's eye view of the prototype

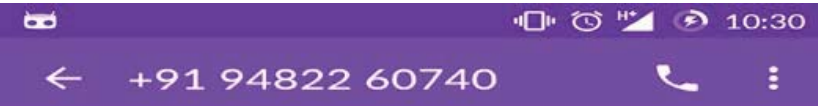

2. Thank you for Shopping with

Bosch. Your order has been

dispatched. you can now live

track Maxima Truck KA O1 T

4635

26 Apr. 14:35

Figure.4. SMS received when the order is ready for dispatch. 


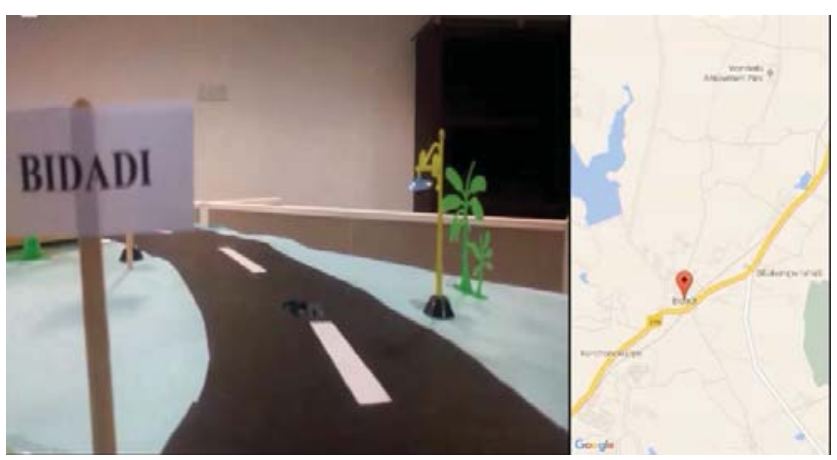

Figure 5.Truck picking up order from Plant 1 along with Live Tracking

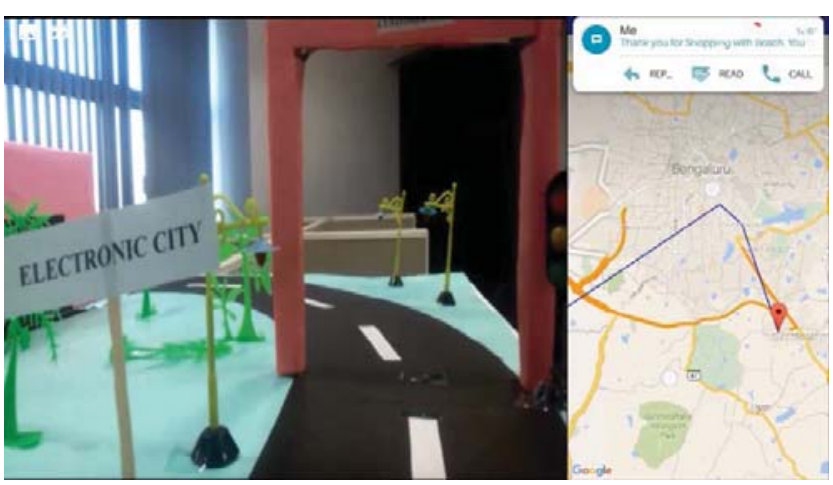

Figure 6.Product delivered at customer location

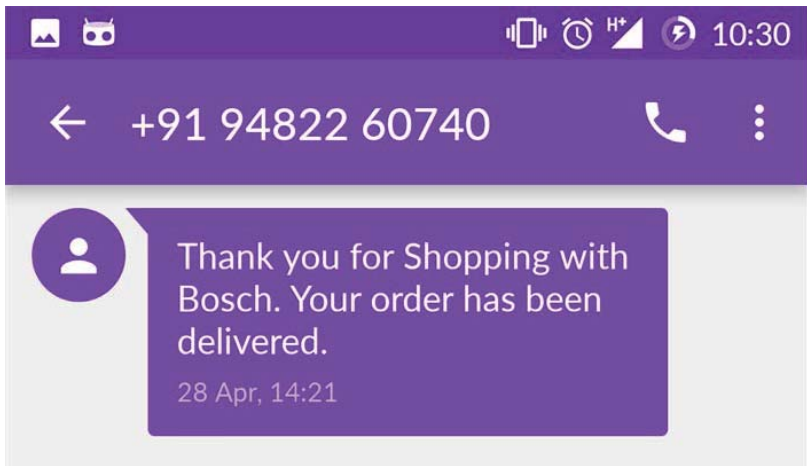

Figure 7 .SMS received when the order is delivered to the customer.

\section{CHALLENGES AND FUTURE SCOPE}

The most decisive challenge is the internet connectivity. For the truck to be tracked to and to control the devices, a gsm module is required to be installed in it. In terrains like Indian, where the manufacturer is away from the city, the internet connectivity is not as strong and developed as it is in western countries. There is also a problem of access permissions. It is also possible that the gsm devise is gets stolen there by deviating from the problem statement in all.

Above challenges can be solved by enabling the necessary requirements such as internet connectivity and access permissions. The GPS of phone is enabled to high accuracy mode to prevent the application from pointing to inaccurate locations. The gsm modules are sealed and placed within pallets, so it is difficult to find its location and are hidden from theft. 


\section{CONCLUSION}

Logistics in I4.0 (LI0) is a very helpful application to track all orders and inform them about the changes and in addiction to a device that monitors truck temperature and humidity can suggest possible outlets on perishable products.

The project brings together under one roof the user, the producer, the home logistics, and the smart warehouse. It automatizes the end to end need of the production phase and enables live tracking of the truck and the products with it. The application is one touch and easy to use. The entire concept aims at decreasing human intervention in the manufacturing process and the consequent errata. The consumer gets to plan in advance his requirements with the data analytics provided through the application. Logistics in I4.0 includes modules such as smart parking, eradicating the conventional and tiring barcode scanning for product identification.

The product can be expanded and enhanced by providing the customer an update about his product purchase history, automatic suggestion and recommendation, increase the production automatize ordering process. Increase security by encrypted lock mechanism and tracking the stolen products.

\section{REFERENCES}

[1] Bassi, Alessandro, "Enabling Things to Talk: Designing IoT Solutions with the IoT Architectural Reference Model", 2013, Vol. 1 No. $1,1-12$.

[2] X. Fang et. Al. , "Smart transport- The new and improved power grid: A Survey”, IEEE Communication Surveys \& Tutorials, , 2015, Vol. 14, No.4, 944-980.

[3] Wei, L. Wei, L. Xin, L. "Design and implement on smart home system", In proceedings of the Fourth International Conference on Intelligent Systems Design and Engineering Applications, 2013.

[4] S. Jain, A. Vaibhav and L. Goyal, "Raspberry pi based interactive home automation system through e-mail," in 2014 International Conference on Reliability, Optimization and Information Technology, (2014) February 6-8, pp. 277-280.

[5] C. Perera, P. P. Jayaraman, A. Zaslavsky, D. Georgakopoulos, and P. Christen, "Sensor discovery and configuration framework for the internet of things paradigm," in Internet of Things (WF-IoT), 2014 IEEE World Forum on. IEEE, 2014, pp. 94-99.

[6] S. Lee, D. Yoon, and A. Ghosh, "Intelligent parking lot application using

[7] Wireless sensor networks," in Proc. Int. Symp. Collab. Technol. Syst., Chicago, May 19-23, 2008, pp. 48-57.

[8] P. Bellavista, G. Cardone, A. Corradi, and L. Foschini, "Convergence of MANET and WSN in IoT urban scenarios," IEEE Sens. J., vol. 13 , no. 10 , pp. $3558-3567$, Oct. 2013

[9] R. N. Murty et al., "CitySense: An urban-scale wireless sensor network and testbed," in Proc. IEEE Technol. Homeland Sec. (HST'08), Waltham,MA, USA, May 2008, pp. 583-588.

[10] Industry 4.0: Towards future industrial opportunities and challenges Keliang Zhou; Taigang Liu; Lifeng Zhou Fuzzy Systems and Knowledge Discovery (FSKD), 2015 12th International Conference Year: 2015

[11] S. Qi Y. Zheng ; M. Li ; L. Lu ; Y. Liu Secure and Private RFID-Enabled Third-Party Supply Chain Systems. IEEE Transactions on Computers (Volume:PP, Issue: 99 ) 04 March 2016

[12] Design and simulation of state-of-art ZigBee transmitter for IoT wireless devicesT. Elarabi; V. Deep; C. K. Rai2015 IEEE International Symposium on Signal Processing and Information Technology (ISSPIT) 2015.

[13] Implementation and analysis of pluggable Android applications A. Singhai; R. S. Ramanujam; J. Bose; V. Kumari Signal Processing, Informatics, Communication and Energy Systems (SPICES), 2015 IEEE International Conference

[14] The working principle of an Arduino Y. A. Badamasi Electronics, Computer and Computation (ICECCO), 2014 11th International Conference Year: 2014 